\title{
AN ATTEMPT TO CLASSIFY THE DIAGNOSTIC ALLERGENS OF CONTACT HYPERSENSITIVITY IN DENTAL BIOMATERIALS
}

\author{
Iliyana Stoeva \\ Department of Allergology, Physiotherapy and Clinical radiology, \\ Faculty of Dental Medicine, Medical University - Plovdiv, Bulgaria
}

\begin{abstract}
There is a wide range of dental biomaterials with varied and complex composition. There are too many sensibilizing substances in dental materials and a careful study of their composition is necessary for choose the appropriate contact allergens in cases of suspicion of contact hypersensitivity to dental materials. We attempt to facilitate the diagnostic of contact allergy due to dental biomaterials by classifying the basic sensitizing components in different groups of dental biomaterials.
\end{abstract} diagnostic

Key words: dental materials, contact hypersensitivity,

The diagnostic of contact hypersensitivity to dental materials in Bulgaria was carrying out for many decades with allergens made from commercial products $(2,3)$. A certain difficulty in the diagnostic process was the wide variety of commercial products and the impossibility to prepare the diagnostic allergens opportunely $(4,5)$. A serious problem was the low concentration of some substances in the material, which was the cause of missing of such substances in the diagnostic allergen made on the base of commercial product $(1,4)$. It is a real cause to read false negative results in cases of sensibilization to those substances.

We made a large study on the composition of dental materials using monographs on dental biomaterials, information of data safety material sheets and correspondence with manufactures. We succeeded to classify the basic standardized diagnostic allergens for different groups of dental materials and we propose that information at Tables 1, 2, 3, 4 and 5 .

Table 1. Allergens for diagnostics of contact hypersensitivity to restorative composite materials.

\begin{tabular}{|l|c|}
\hline ALLERGENS & Conc./pet.\%(w/w) \\
\hline Methacrylate monomers: & 2.0 pet \\
1. Bis-GMA - Bisphenol A diglycidyl ether methacrylate; & \\
[2,2-bis-(4-(2-hydroxy-3-methacryloxypropoxy)phenyl)propane]; & 2.0 pet \\
2. Bis-EMA - Bisphenol A polyethylene glycol diether dimethacrylate; & \\
[2,2-bis-(4-(2-methacryloxyethoxy)phenyl)propane]; & 2.0 pet \\
3. UDMA - Urethane dimethacrylate; & 2.0 pet \\
4. TEGDMA - Triethyleneglycol dimethacrylate; & 2.0 pet \\
5. EGDMA - Ethyleneglycol dimethacrylate & \\
\hline Initiators: & 1.0 pet \\
1. CQ - Camphoroquinone; & 1.0 pet \\
2. BP - Benzoyl peroxide & \\
\hline Activators: & 0.2 pet \\
1. DMAEMA - N,N-dimethylaminoethyl methacrylate & 5.0 pet \\
2. DMPT - N,N-Dimethyl-4-toluidine & \\
\hline Inhibitors: & 1.0 pet \\
1. MEHQ - Methylhydroquinone & \\
\hline UV-absorbers: & 10.0 pet \\
1. HMBP - 2-hydroxy-4-methoxy benzophenone; & 1.0 pet \\
2. Tinuvin P - 2-(2-hydroxy-5 methylphenyl) benzotriazole & \\
\hline
\end{tabular}


Tab. 2. Allergens for diagnostics of contact hypersensitivity to dental amalgam.

\begin{tabular}{|l|c|}
\hline ALLERGENS & Conc./pet. $\%(\mathbf{w} / \mathbf{w})$ \\
\hline 1. $\mathbf{H g}$ - Mercury & $0.5 \mathrm{pet}$ \\
2. Ag - Silver nitrate & $1.0 \mathrm{aq}$ \\
3. $\mathbf{S n}$ - Tin & $50.0 \mathrm{pet}$ \\
4. $\mathbf{C u}$ - Copper sulfate & $2.0 \mathrm{pet}$ \\
$\mathbf{5 . Z n}-$ Zinc chloride & 2.0 pet \\
\hline
\end{tabular}

Tab. 3. Allergens for diagnostics of contact hypersensitivity to dental acrylate plastics.

\begin{tabular}{|c|c|}
\hline ALLERGENS & Conc./pet.\%(w/w) \\
\hline $\begin{array}{l}\text { Methacrylate monomers: } \\
\text { 1. MMA - Methyl methacrylate; } \\
\text { 2. EGDMA - Ethyleneglycol dimethacrylate; } \\
\text { 3. UDMA - Urethane dimethacrylate }\end{array}$ & $\begin{array}{l}2.0 \text { pet } \\
2.0 \text { pet } \\
2.0 \text { pet }\end{array}$ \\
\hline $\begin{array}{l}\text { Initiator of polymerization: } \\
\text { 1. BP - Benzoylperoxide } \\
\text { Possible component in initiator system: } \\
\text { 1. Cu - Copper sulfate }\end{array}$ & $\begin{array}{l}1.0 \text { pet } \\
2.0 \text { pet }\end{array}$ \\
\hline $\begin{array}{l}\text { Initiator for visible light dental acrylic composite materials: } \\
\text { 1. CQ - Camphoroquinone }\end{array}$ & 1.0 pet \\
\hline $\begin{array}{l}\text { Accelerator for polymerization: } \\
\text { 1. DMPT - N,N-dimethyl-p-toluidine }\end{array}$ & 5.0 pet \\
\hline $\begin{array}{l}\text { Stabilizer and antioxidant: } \\
\text { 1. HQ - Hydroquinone }\end{array}$ & 1.0 pet \\
\hline $\begin{array}{l}\text { Plastificator: } \\
\text { 1. Dibutyl-phthalate }\end{array}$ & 5.0 pet \\
\hline $\begin{array}{l}\text { Product of oxidation of MMA: } \\
\text { 1. Formaldehyde }\end{array}$ & $1.0 \mathrm{aq}$ \\
\hline
\end{tabular}

Tab. 4. Allergens for diagnostics of contact hypersensitivity to metals in dental alloys.

\begin{tabular}{|c|c|}
\hline ALLERGENS & Conc./pet.\%(w/w) \\
\hline $\begin{array}{l}\text { No noble metals: } \\
\text { 1. } \mathbf{N i}-\text { Nickel sulfate } \\
\text { 2. Co-Cobalt chloride } \\
\text { 3. } \mathbf{C r}-\text { Potassium dichromate } \\
\text { 4. Mo- Molybdenum } \\
\text { 5. Al - Aluminum chloride hexahydrate } \\
\text { 6. Mn - Manganese (II) chloride } \\
\text { 7. Ti - Titanium } \\
\text { 8. } \mathbf{T i}-\text { Titanium oxalate } \\
\text { 9. } \mathbf{Z n}-\text { Zinc chloride } \\
\text { 10. } \mathbf{C u} \text { - Copper sulfate }\end{array}$ & $\begin{array}{l}5.0 \text { pet } \\
1.0 \text { pet } \\
0.5 \text { pet } \\
5.0 \text { pet } \\
2.0 \text { pet } \\
2.0 \text { pet } \\
10.0 \text { pet } \\
5.0 \text { pet } \\
2.0 \text { pet } \\
2.0 \text { pet }\end{array}$ \\
\hline $\begin{array}{l}\text { Noble metals: } \\
\text { 1. Au - Goldiumthiosulphate } \\
\text { 2. Ag - Silver nitrate } \\
\text { 3. } \mathbf{P d} \text { - Palldium chloride }\end{array}$ & $\begin{array}{l}2.0 \mathrm{pet} \\
1.0 \mathrm{aq} \\
2.0 \mathrm{pet}\end{array}$ \\
\hline
\end{tabular}


${ }^{1}$ The allergen is appropriate for suspicion of contact allergy to dental implants.

2 The allergen is appropriate for suspicion of contact allergy to titanium in dental alloys.

Tab. 5. Allergens for diagnostics of contact hypersensitivity to materials for endodontic fillings.

\begin{tabular}{|l|c|}
\hline ALLERGENS & Conc./pet.\%(w/w) \\
\hline 1. Eugenol & $2.0 \mathrm{pet}$ \\
2. Formaldehyde & $1.0 \mathrm{aq}$ \\
3. Balsam Peru & $25.0 \mathrm{pet}$ \\
4. Epoxy resins & $1.0 \mathrm{pet}$ \\
\hline
\end{tabular}

\title{
CONCLUSION
}

Such classification of contact allergens assure with relatively little number of diagnostic allergens to assess the reactivity of the patients to a large number commercial dental biomaterials.

\section{REFERENCES:}

1. Ivanova, I. Intraoral contact hypersensitivity to prosthodontic biomaterials. Dissertation 2008. (in Bulgarian)

2. Kisselova, A. Dental allergology and Focal diagnostic. Monograph, publisher "Gutenberg", Sofia, 2001, p.327. (in Bulgarian)
3. Petrunov, B., V. Dimitrov, A. Kisselova. Clinical immunology, clinical allergology, clinical dental allergology. Book, publisher "'Arso", Sofia, 2009.

4. Stoeva, I., R. Velikova, A. Kisselova. An attempt to improve diagnostics of contact allergy due to dental composite materials. Problems of Dental Medicine 2010; XXXVI (2):80-87. (in Bulgarian)

5. Stoeva, I. Practical guide of clinical dental allergology. Publisher ''Medical University-Plovdiv", 2011, p.93. (in Bulgarian)

\author{
Adrress for correspondence: \\ Iliyana Stoeva \\ Department of Allergology, Phisiotherapy and Clinical Radiology, Faculty of \\ Dental Medicine, Medical University - Plovdiv \\ 3, Hristo Botev Blvd., 4000 Plovdiv, Bulgaria \\ E-mail: stoeva_iliana@abv.bg
}

